\title{
Molecular features of water films created with bubbles at silica surfaces
}

\section{$1 \quad$ Xuming Wang PhD}

Research Professor, Department of Metallurgical Engineering, College of Mines and Earth Sciences, University of Utah, Salt Lake City, UT, USA

2 Xihui Yin PhD\#

Former Graduate Student, Department of Metallurgical Engineering, College of Mines and Earth Sciences, University of Utah, Salt Lake City, UT, USA

3 Jakub Nalaskowski PhD

Senior Research Scientist, Cabot Microelectronics, Aurora, IL, USA
4 Hao Du PhD

Professor, National Engineering Laboratory for Hydrometallurgical Cleaner Production Technology, Institute of Process Engineering, Chinese Academy of Sciences, Beijing, China

5 Jan D. Miller PhD*

Ivor D. Thomas Distinguished Professor, Department of Metallurgical Engineering, College of Mines and Earth Sciences, University of Utah, Salt Lake City, UT, USA
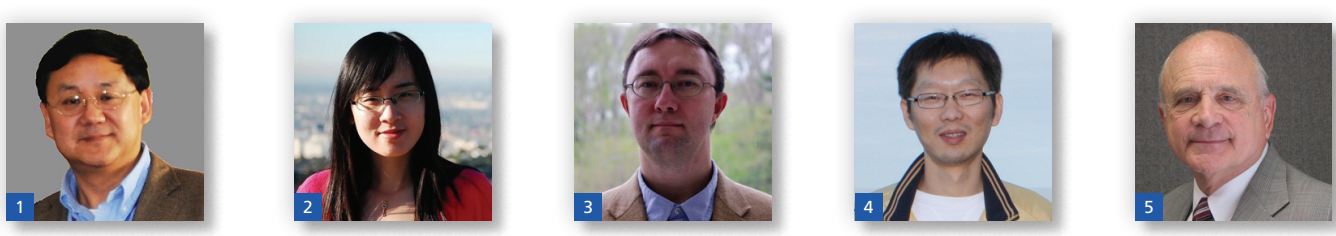

Sum frequency vibrational spectroscopy (SFVS) spectra indicate that a very ordered water structure exists in the stable water film at a hydrophilic silica surface during contact with a bubble and that the extent of hydrogen bonding increases with an increase in contact pressure. In contrast, the SFVS spectra of water at a hydrophobic silica surface show a lack of hydrogen bonding and are characterized by a distinct absorption at about $3700 \mathrm{~cm}^{-1}$, similar to the spectrum of the air/water interface. These results suggest the presence of a water exclusion zone at the hydrophobic surface, as supported by X-ray reflectivity measurements reported in the literature, by AFM images, and by results from molecular dynamics simulations. Of course, the water film at the hydrophobic surface is unstable with film thinning and rupture upon bubble contact. Under these circumstances, it is shown that an attractive van der Waals force between a bubble and the hydrophobic surface can be expected when the water exclusion zone is taken into consideration. As the thickness of the water exclusion zone increases to a thickness corresponding to the size of nanobubbles, the calculated attractive van der Waals force increases. This analysis may help to explain the so-called 'short-range' and 'long-range' hydrophobic forces.

\section{Introduction}

The interaction of bubbles with solid and liquid particles in aqueous systems is a fundamental phenomenon of interest in many areas of technology. These interactions are important in the development of improved flotation technologies for the recovery of valuable minerals, for water treatment, for wastepaper recycle, and so on. Flotation is accomplished by the attachment of air bubbles at the surface of hydrophobic particles and their separation from suspension, as practiced in the processing of mineral and energy resources. Attention has been given to the physics of bubble interactions at surfaces, including the bubble approach to the surface, the formation of the intervening water film, the kinetics of film thinning, the critical film thickness, the film's stability, the interaction forces and the disjoining pressure. ${ }^{1-10}$ All these studies indicate that the structures of these water films differ from those of water in the bulk phase.

It has been established for silica surfaces that water films between a negatively charged bubble and the negatively charged silica surface are on the order of $100 \mathrm{~nm}$ in thickness. The films are stable at clean hydrophilic silica surfaces and metastable at methylated hydrophobic silica surfaces. ${ }^{1}$ These pioneering studies have 
been extended for metastable films to determine film stability including, drainage, rupture and formation of the three-phase line of contact. ${ }^{4,5,8}$ Although considerable study has been given to the physics of film stability between a bubble and a surface, little information is available regarding the chemistry of the water film and the chemical reasons for film stability at a hydrophilic surface and instability at a hydrophobic surface. Now, it has been shown that important chemical information on hydrogen bonding of interfacial water molecules as related to these water films can be obtained by sum frequency vibrational spectroscopy (SFVS) and that consideration of a water exclusion zone at the hydrophobic surface results in an attractive van der Waals force between the bubble and the hydrophobic surface.

\section{Experimental procedure for SFVS}

A hemicylindrical prism of fused silica $\left(\mathrm{SiO}_{2}\right.$; Almaz Optics, Marlton, NJ) serves as the solid substrate for the SFVS experiments. For the hydrophilic silica, the silica substrate is cleaned in a solution of $70 \% 18 \mathrm{M}$ sulphuric acid and $30 \% \mathrm{H}_{2} \mathrm{O}_{2}$ for $4 \mathrm{~h}$ followed by washing with water at room temperature. The prism is then rinsed thoroughly with DI water, dried under a flow of nitrogen and subsequently argon plasma cleaned for $10 \mathrm{~min}$.

In order to prepare the hydrophobic silica surface (advancing water contact angle $105^{\circ}$ ), the silica substrate is cleaned with the same procedure and then the surface is modified with octadecyltrichlorosilane (OTS). ${ }^{11}$ To make the surface hydrophobic, the cleaned prism is placed into a $0 \cdot 1 \%$ OTS benzene solution for $\sim 15 \mathrm{~h}$. Then the prism is taken from the solution and rinsed three times using $\mathrm{CHCl}_{3}$ to remove residual OTS from the prism. After that, the prism is dried in an oven for $4 \mathrm{~h}$ at $100^{\circ} \mathrm{C} .{ }^{11}$

A water sample cell (Figure 1) with an air bubble screw holder is used to conduct the SFVS experiments on a sum frequency vibrational

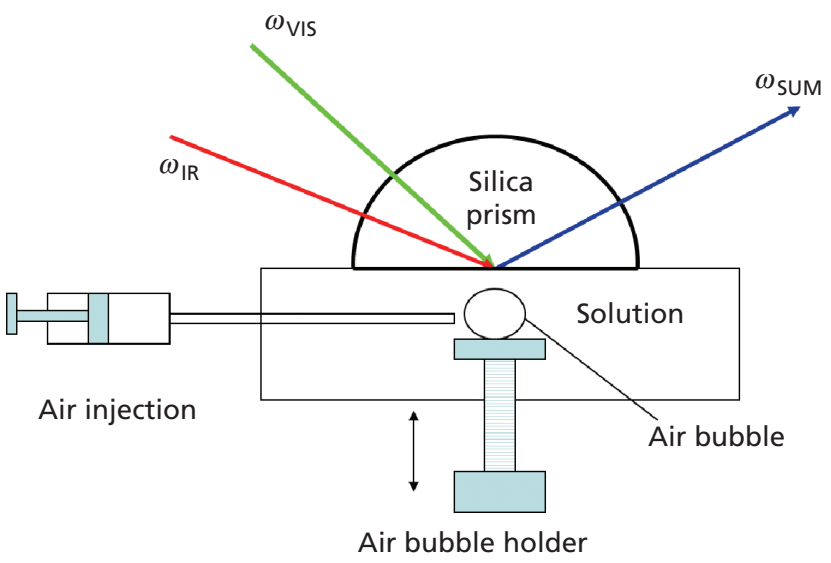

Figure 1. Experimental setup to measure sum frequency spectra of water films created with a bubble at hydrophilic and hydrophobic silica surfaces spectrometer (EKSPLA, Ltd., Vilnius, Lithuania, Baltic States). The laser system has been described in previous publications, ${ }^{12}$ so a description is not provided here. The spectra presented were taken under ssp polarization conditions for the three beams (s-polarized sum-frequency, s-polarized visible and p-polarized infrared). Data were collected at $4 \mathrm{~cm}^{-1}$ increments, and each point is the average of 30 laser shots. All spectra were recorded at a constant temperature of $23^{\circ} \mathrm{C}$. DI water was used in all experiments.

\section{Results}

Water films at both hydrophilic and hydrophobic silica surfaces were examined by SFVS, and the spectral results are discussed in terms of hydrogen bonding of interfacial water molecules. Repulsive electrostatic and van der Waals forces, as well as strong hydrogen bonding of ordered water molecules, account for film stability for the bubble at the hydrophilic silica surface.

In contrast, the attraction between the bubble and hydrophobic surface, and the corresponding water film instability, is explained based on the lack of hydrogen bonding in the water film and the presence of a water exclusion zone at the hydrophobic surface.

\subsection{Hydrophilic silica surface}

Using the experimental setup shown in Figure 1, the SFVS spectrum of the interface for a water film separating a bubble from the hydrophilic silica surface is determined for a buoyant bubble and for a bubble pressed to the silica surface by a screw adjustment of the bubble holder. As a result of pressing the bubble at the silica surface, there is a sequential increase in bubble deformation, from an undeformed spherical cap to a disc-shaped bubble, and concurrently the thickness of the water film is diminished. However, the water film remains stable under these conditions.

The spectral results are presented in Figure 2. For the undeformed bubble, the spectrum of the interfacial water is similar to bulk water at a hydrophilic silica surface and is characterized by three bands, the ice-like peak at $3176 \mathrm{~cm}^{-1}$ corresponding to complete tetrahedral coordination, the liquid water band at $3408 \mathrm{~cm}^{-1}$ corresponding to incomplete tetrahedral coordination and the free $\mathrm{OH}$ band at $3685 \mathrm{~cm}^{-1}$. As the bubble is placed in contact and deformed under gravity, the thickness of the water film decreases, and the spectrum changes significantly. The free $\mathrm{OH}$ band at $3685 \mathrm{~cm}^{-1}$ is eliminated, and the intensity of the ice-like band at $3176 \mathrm{~cm}^{-1}$ becomes more intense. When the bubble is pressed further (forced contact), greater deformation occurs, the water film becomes thinner, and the icelike band at $3176 \mathrm{~cm}^{-1}$ becomes even more intense. The elimination of the free $\mathrm{OH}$ band at $3685 \mathrm{~cm}^{-1}$ suggests that hydrogen bonding of water molecules with silica surface sites and with each other has been increased, as expected for this hydrophilic surface.

It is important to note that the band intensity for ice-like water increases, also suggesting a significant increase in well-ordered 
hydrogen bonding of water molecules at the surface. SFVS band intensities for water rarely exceed 5 units, as is the case for the silica-water system in Figure 2. With increased pressure (forced contact) and diminished film thickness, the intensity of the icelike peak increases by an order of magnitude to 50 units. Under these circumstances, film rupture does not occur, the film is stable and the SFVS results demonstrate the chemistry associated with hydrophilic surfaces, the stability of the water film and the lack of flotation of such hydrophilic particles, since bubble attachment does not occur. The AFM and molecular dynamics simulations (MDS) results on the viscosity of interfacial water reported in the literature support this analysis. ${ }^{13}$

These results clearly show that the stability of water films, such as those formed at a hydrophilic silica surface, is explained by the hydrogen bonding of water molecules at surface sites, leading to complete tetrahedral coordination, which is common to the ice-like structure of water. Furthermore, hydrogen bonding and order of water molecules at the silica surface increase with forced contact, to resist rupture of the water film. Such structural considerations as well as repulsive electrostatic and van der Waals forces account for water film stability at the hydrophilic silica surface.

\subsection{Hydrophobic silica surface}

In the case of the hydrophobic OTS-coated silica surface (a water contact angle of $105^{\circ}$ ), water films are unstable. Rupture and film displacement occur spontaneously with little force even though the silica surface and bubble surface are both negatively charged. ${ }^{1}$ Because of this instability, the dynamic change in hydrogen

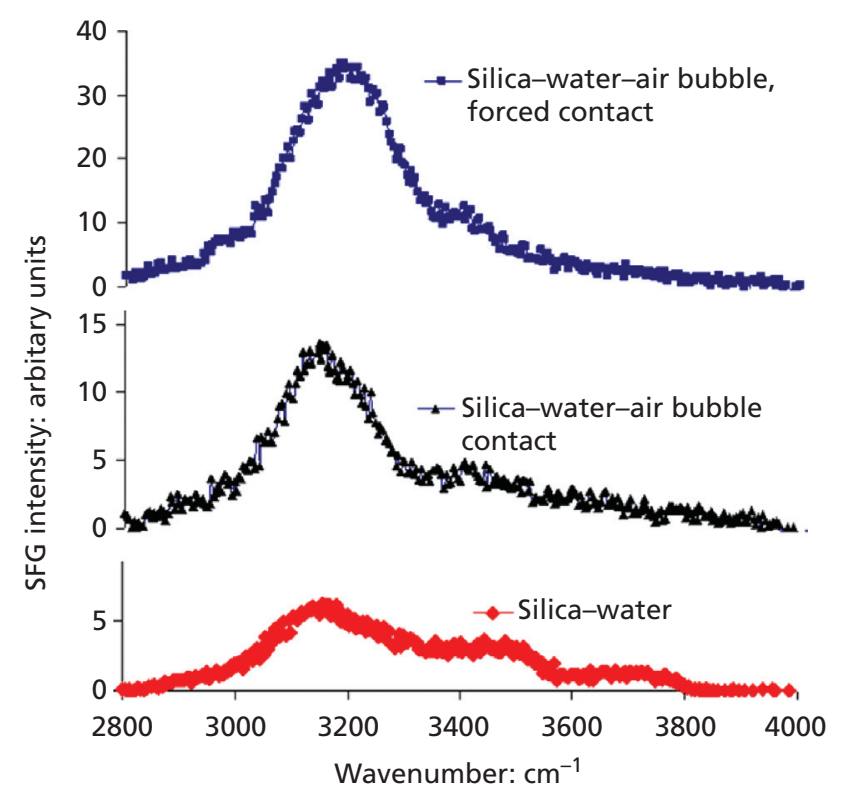

Figure 2. Sum frequency vibrational spectroscopy spectra for air bubbles at the hydrophilic silica surface bonding of the water film at a hydrophobic surface during rupture has not been measured. Nevertheless, it can be seen from Figure 3 that, prior to bubble contact, the water film spectrum is similar to the spectrum of bulk water at a hydrophobic OTS silica surface.

The water spectrum at a hydrophobic surface is distinctly different from the water spectrum at a hydrophilic surface. ${ }^{14}$ As shown in Figure 3, the major band is the free $\mathrm{OH}$ vibration at $3676 \mathrm{~cm}^{-1}$. Unlike other results, ${ }^{15}$ there is little evidence of hydrogen bonding, perhaps related to well-ordered OTS and a lower level of surface polarity. Note the absence of bands at $\sim 3200$ and $3400 \mathrm{~cm}^{-1}$ (the peaks at 2876 and $2940 \mathrm{~cm}^{-1}$ are from $\mathrm{CH}$ stretching of OTS molecules). Of course with slight pressure, the water film, with no evidence of hydrogen bonding at the surface, becomes unstable. Rupture occurs, followed by bubble attachment and, under these circumstances, the water signal at $3676 \mathrm{~cm}^{-1}$ is eliminated, as shown in Figure 3. It appears that the SFVS spectrum remains unchanged until the moment of rupture. After attachment, there is no evidence of residual water, and the spectrum is similar to the spectrum for air at the hydrophobic OTS silica surface (Figure 3).

\section{Discussion}

Water at a hydrophobic surface is distinguished in the SFVS spectrum by the strong band at $\sim 3700 \mathrm{~cm}^{-1}$ corresponding to the free $\mathrm{OH}$ vibration, from water molecules that are not $\mathrm{H}$-bonded. The water spectrum at the hydrophobic surface is similar to the SFVS spectrum for the air-water interface. ${ }^{14}$ These results suggest the presence of a water void, or water exclusion zone, at the hydrophobic

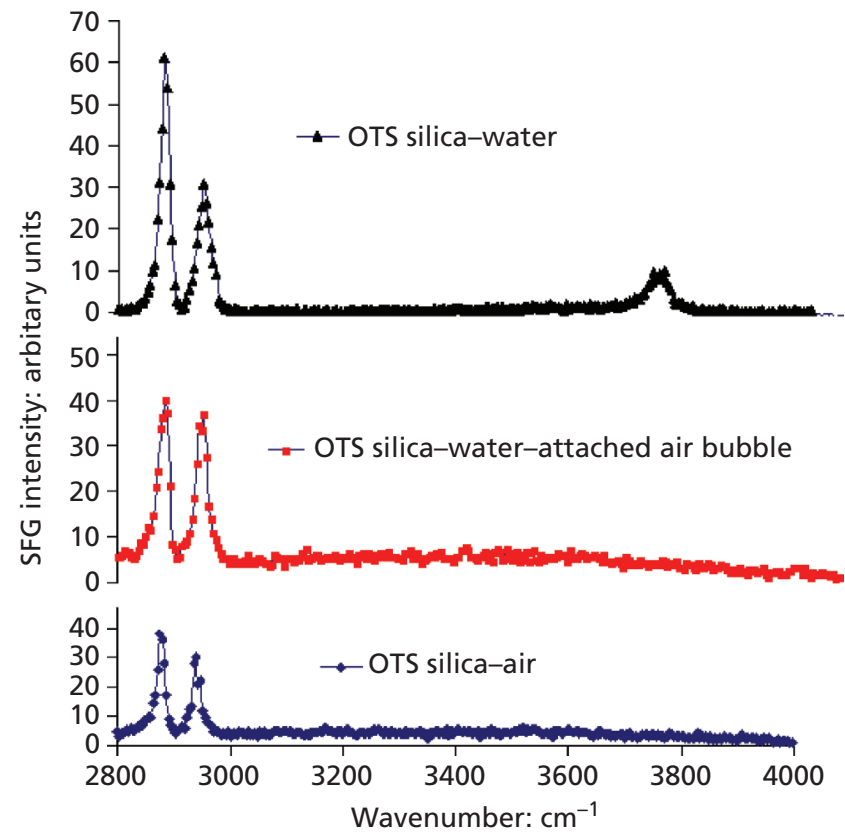

Figure 3. Sum frequency vibrational spectroscopy spectra for the hydrophobic octadecyltrichlorosilane silica surface 


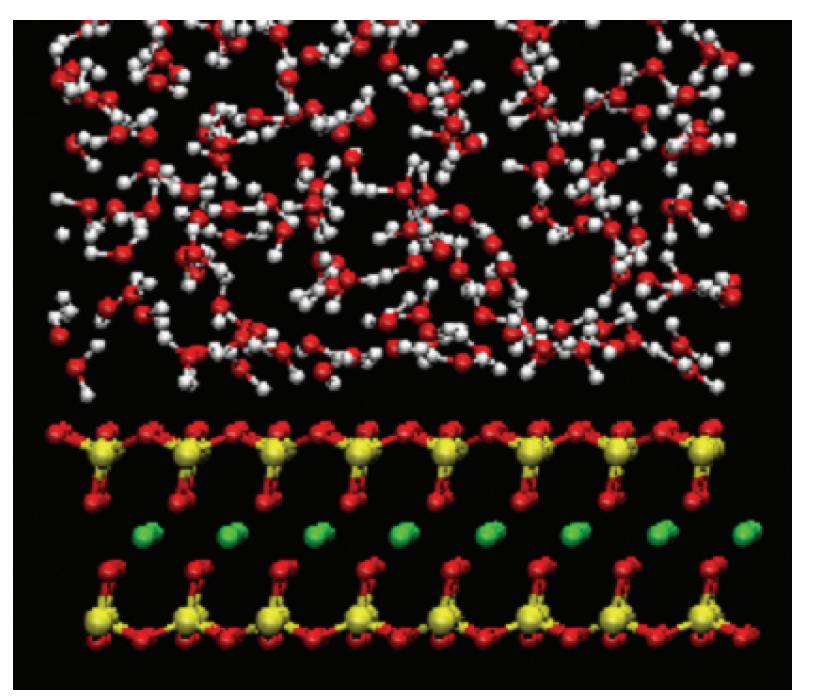

Figure 4. Molecular dynamics simulations snapshot of equilibrated water at the hydrophobic talc basal plane surface (left) and corresponding interfacial water density distribution function (right).

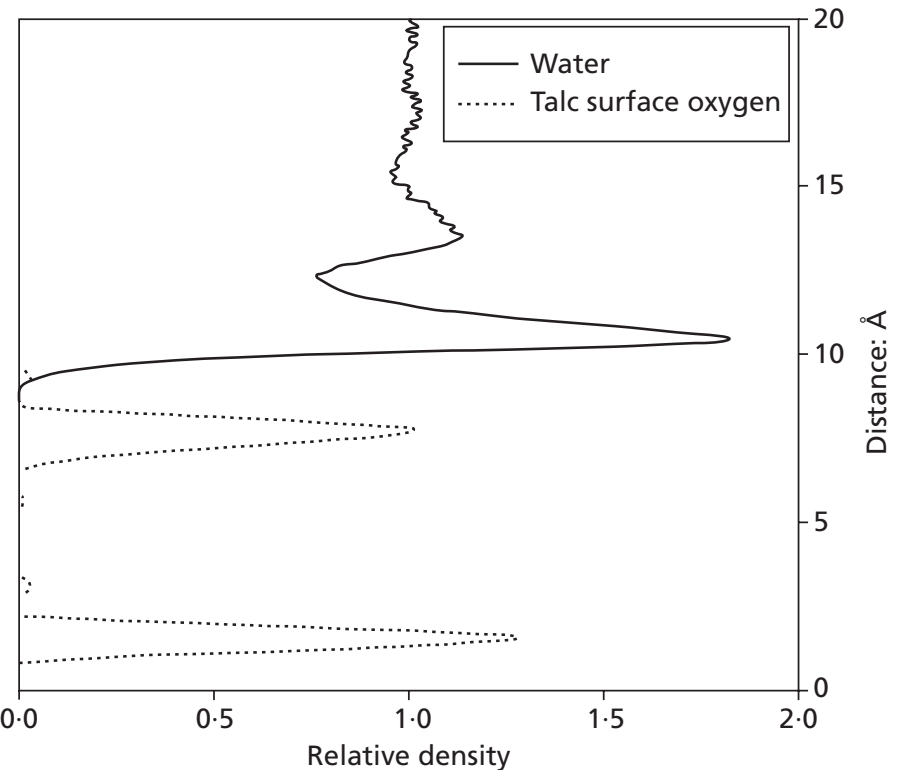

The colour representations are as follows: red (dark colour), oxygen atoms; white (light colour), hydrogen atoms; yellow, silicon atoms; and green, magnesium atoms ${ }^{17}$
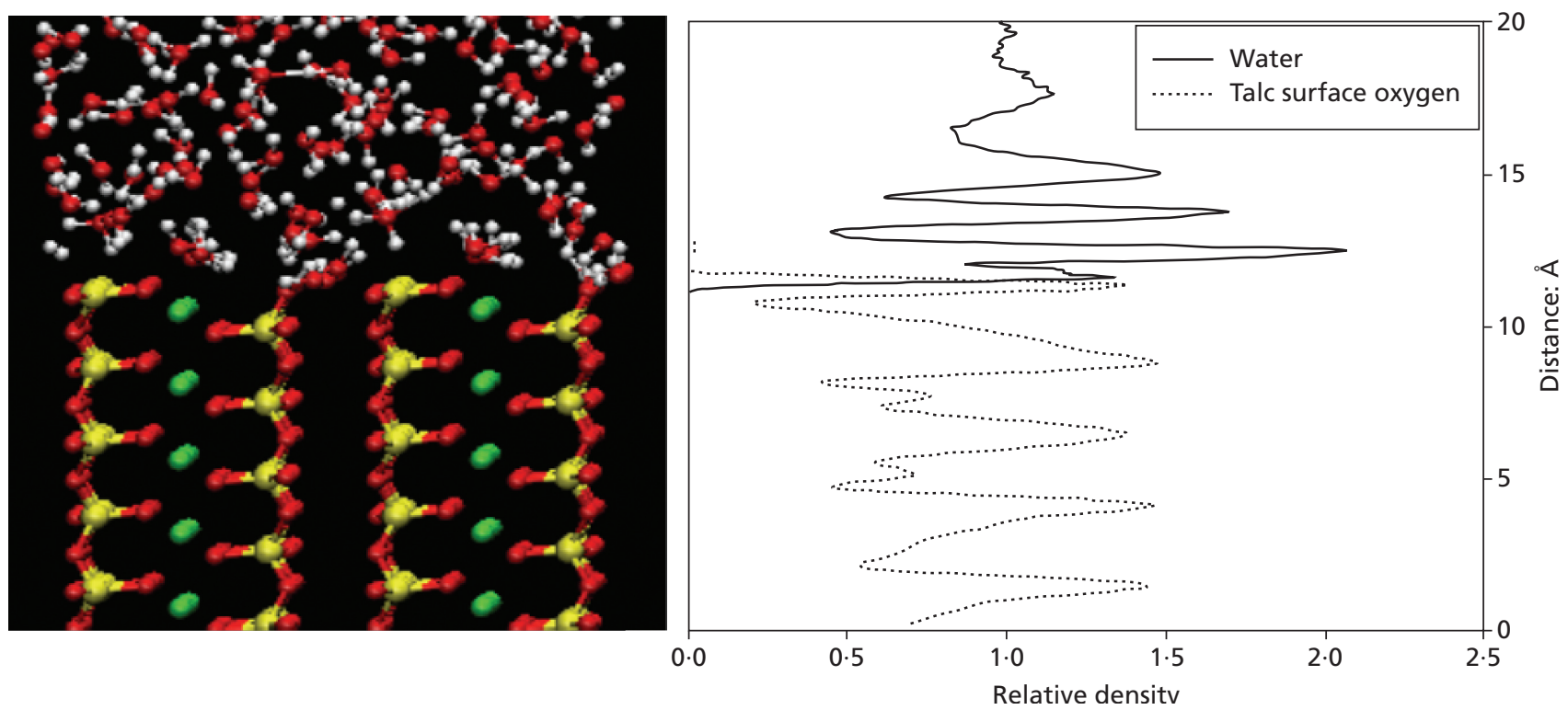

Figure 5. Molecular dynamics simulations snapshot of equilibrated water at the hydrophilic talc edge surface (left) and corresponding interfacial water density distribution function (right). The colour

representations are as follows: red (dark colour), oxygen atoms; white (light colour), hydrogen atoms; yellow, silicon atoms; and green, magnesium atoms ${ }^{17}$

surface, as supported by X-ray reflectivity measurements reported in the literature ${ }^{16}$ and which has been demonstrated for water at many different hydrophobic surfaces by MDS. ${ }^{17}$ For example, see the MDS of water at the hydrophobic basal plane talc surface presented in Figure 4. Note the water exclusion zone. In fact, the
MDS results show the thickness of the water exclusion zone to be about $3.5 \AA$, the same as that determined from X-ray reflectivity measurements. In contrast, the MDS of the hydrophilic talc edge surface presented in Figure 5 shows that water bonds directly with the edge surface. Clearly the water exclusion zone is only present at 
Surface Innovations

Volume 3 Issue SI1
Molecular features of water films created

with bubbles at silica surfaces

Wang, Yin, Nalaskowski, Du and Miller the hydrophobic basal plane surface of talc. At the hydrophilic edge surface of talc, water is readily accommodated at surface sites, and no exclusion zone is found.

In addition to indicating the water exclusion zone, MDS results also provide information regarding hydrogen bonding. For example, Figure 6 shows the distribution of hydrogen bonds along the surface normal for a hydrophobic pyrophyllite surface and hydrophilic quartz surface. The pyrophyllite surface has a crystalline structure similar to talc. The only difference is that pyrophyllite has aluminium in its octahedral layer, whereas talc has magnesium. In MDS, with the simple point charge (SPC) water model, a water molecule in the bulk liquid has on average about 3.2-3.3 H-bonds. As shown in Figure 6, it may be noticed that, at the pyrophyllite surface, there is no hydrogen bond in the interfacial region extending to a distance of $2 \AA$, due to the exclusion of water, revealing the surface's hydrophobic character. In contrast, at the hydrophilic quartz surface, the interfacial water molecules are tightly bonded with the surface oxygen atoms. The number of hydrogen bonds in the interfacial region for the hydrophilic quartz surface is about 4, suggesting that almost all the water molecules are tetrahedrally bonded. These results are in good agreement with the SFVS results.

Finally, extensive experimental results from AFM measurements ${ }^{18,19}$ and results from other experiments ${ }^{20}$ reveal the presence of a gas phase at hydrophobic surfaces. This gas phase is frequently observed as flattened nanobubbles. Therefore, it is evident that the hydrophobic surface state must include a water exclusion zone which may vary in thickness from less than one nanometre to tens of nanometres, the size of nanobubbles. In this regard, the analysis of the attractive force between a bubble and a hydrophobic

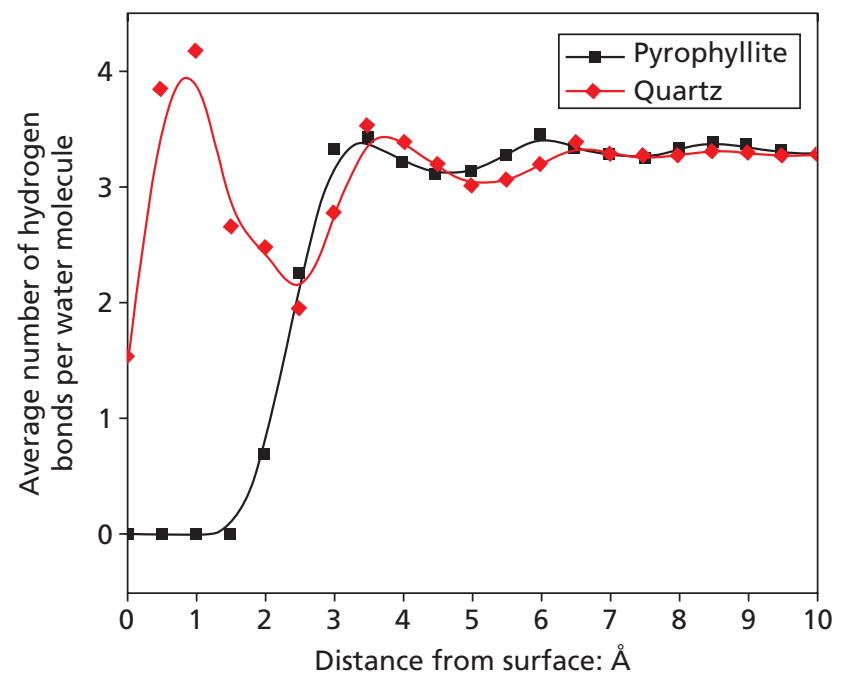

Figure 6. Comparison of the average number of hydrogen bonds for each water molecule at a hydrophobic pyrophyllite surface and a hydrophilic quartz surface surface should take into account the presence of a void space (gas film) or water exclusion zone, extending to the dimensions even of nanobubbles.

The general form of the equation that describes the van der Waals force between two surfaces ( $1^{\prime}$ and 1$)$ with adsorbed films $\left(2^{\prime}\right.$ and 2 ) of thickness $T^{\prime}$ and $T$ across the medium 3 is given by Equation 1 according to Israelachvili ${ }^{21}$ where As are corresponding Hamaker constants for interactions between two surfaces across a given medium.

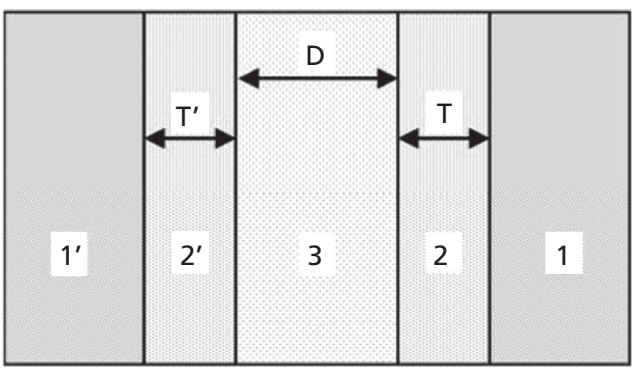

1.

$$
F(D)=-\frac{1}{6 \pi}\left(\begin{array}{l}
\frac{A_{232^{\prime}}}{D^{3}}-\frac{\sqrt{A_{121} \cdot A_{32^{\prime 3}}}}{(D+T)^{3}}- \\
\frac{\sqrt{A_{1^{\prime} 2^{\prime} 1^{\prime}} \cdot A_{323}}}{\left(D+T^{\prime}\right)^{3}}-\frac{\sqrt{A_{1^{\prime} 2^{\prime} 1^{\prime}} \cdot A_{121}}}{\left(D+T+T^{\prime}\right)^{3}}
\end{array}\right)
$$

For the case of an air bubble and a hydrophobic quartz surface covered with an air layer of thickness $\mathrm{T}$ across a water film, the van der Waals equation simplifies to Equation 2.

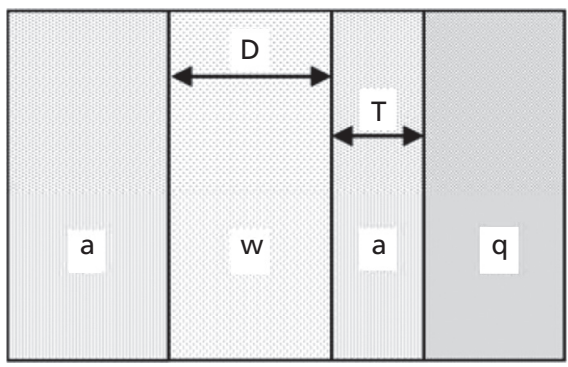

2.

$$
F(D)=-\frac{1}{6 \pi}\left(\frac{A_{\text {awa }}}{D^{3}}-\frac{\sqrt{A_{\text {waw }} \cdot A_{\text {qaq }}}}{(D+T)^{3}}\right)
$$

where $A_{\text {awa }}$ is the air-water-air Hamaker constant $=3.7 \times 10^{-20} \mathrm{~J}$, $A_{\text {waw }}$ is the water-air-water Hamaker constant $=3.7 \times 10^{-20} \mathrm{~J}, A_{\text {qac }}$ is the quartz-air-quartz Hamaker constant $=6.5 \times 10^{-20} \mathrm{~J}$, D is distance and $\mathrm{T}$ is thickness of air layer.

With such a description, the van der Waals force between a bubble and a hydrophobic surface is not repulsive as suggested in the literature. ${ }^{22}$ For example, even when the water exclusion zone is just $2 \AA$ in thickness, an attractive van der Waals force is calculated and observed, as shown in Figure 7. 
Surface Innovations

Volume 3 Issue SI1
Molecular features of water films created with bubbles at silica surfaces

Wang, Yin, Nalaskowski, Du and Miller

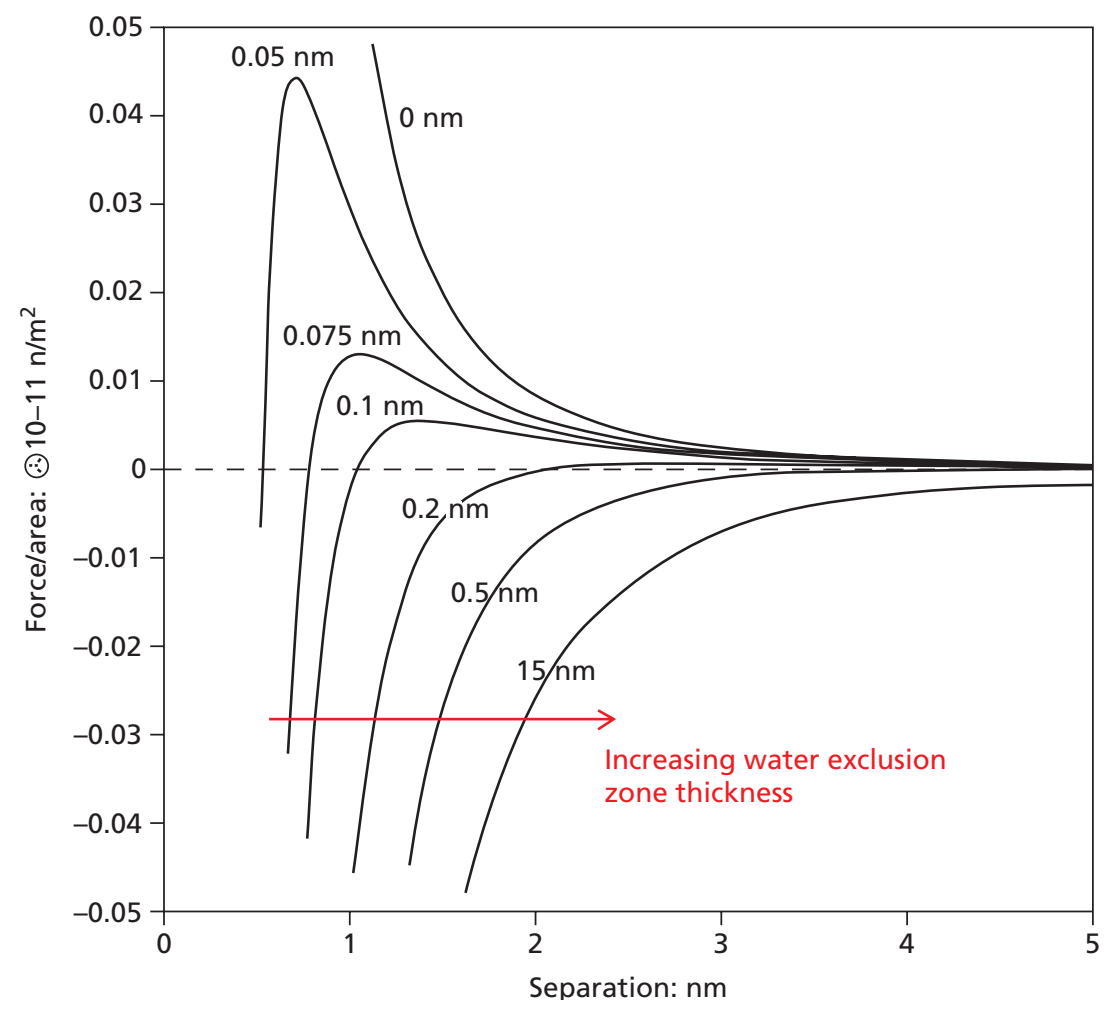

Figure 7. While van der Waals forces are normally thought to be repulsive for bubble attachment at a hydrophobic surface, they became attractive due to the presence of a water exclusion zone. The magnitude of the attractive van der Waals force increases significantly as the water exclusion zone thickness increases

exclusion zone at the hydrophobic surface, which is supported by $\mathrm{X}$-ray reflectivity measurements and by the results from MDS.

Of course, the water film at the hydrophobic surface is unstable with film thinning and rupture upon bubble contact. Under these circumstances, it is shown that an attractive van der Waals force between the bubble and the hydrophobic surface can be expected when the water exclusion zone is taken into consideration. As the thickness of the water exclusion zone increases to a thickness corresponding to the size of nanobubbles, the calculated attractive van der Waals force increases, and this analysis may help to explain the short-range and long-range hydrophobic forces.

\section{Acknowledgements}

Financial support has been provided by the Division of Chemical Sciences, Geosciences, and Biosciences, Office of Basic Energy Sciences of the U.S. Department of Energy through grant number DE-FG-03-93ER14315. The authors thank Xia Zhang for helping in the preparation of the manuscript for publication.

\section{REFERENCES}

1. Blake, T. D.; Kitchener, J. A. Stability of aqueous films on hydrophobic methylated silica. Journal of the Chemical Society, Faraday Transactions 1972, 68(1), 1435-1442. 
2. Butt, H.-J. A technique to measure the force between a colloidal particle in water and a bubble. Journal of Colloid and Interface Science 1994, 166(1), 109-117.

3. Butt, H.-J.; Jaschke, M.; Ducker, W. Measuring surface forces in aqueous solution with the atomic force microscope. Bioelectrochemistry and Bioenergetics 1995, 38(1), 191-201.

4. Hewitt, D.; Fornasiero, D.; Ralston, J. Aqueous film drainage at the quartz/water/air interface. Journal of the Chemical Society, Faraday Transactions 1993, 89(5), 817-822.

5. Hewitt, D.; Fornasiero, D.; Ralston, J. Bubble-particle attachment. Journal of the Chemical Society, Faraday Transactions 1995, 91(13), 1997-2001.

6. Johnson, D. J.; Miles, N. J.; Hilal, N. Quantification of particle-bubble interactions using atomic force microscopy: a review. Advances in Colloid and Interface Science 2006, 127, 67-81.

7. Krasowska, M.; Malysa, K. Wetting films in attachment of the colliding bubble. Advances in Colloid and Interface Science 2007, 134-135, 138-150.

8. Nguyen, A. V.; Drelich, J.; Colic, M.; Nalaskowski, J.; Miller, J. D. Bubbles: interaction with solid surfaces. In Encyclopedia of Surface and Colloid Science, 2nd Edition (Somasundaran, P., (ed.)), New York, NY: Marcel Dekker, 2007, 1-29.

9. Paulsen, F. G.; Pan, R.; Bousfield, D. W.; Thompson, E. V. The dynamics of bubble/particle attachment and the application of two disjoining film rupture models to flotation. Journal of Colloid and Interface Science 1996, 198, 400-410.

10. Scheludko, A.; Toshev, B.; Bogadiev, B. Attachment of particles to a liquid surface, capillary theory of flotation. Journal of the Chemical Society, Faraday Transactions 1 1976, 72, 815-2828.

11. Nalaskowski, J.; Veeramasuneni, S.; Hupka, J.; Miller J. D. AFM measurements of hydrophobic forces between a polyethylene sphere and silica plates-the significance of surface roughness. Journal of Adhesion Science and Technology 1999, 13(12), 1519-1533.

12. Wang, X.; Liu, J.; Du, H.; Miller, J. D. States of adsorbed dodecyl amine and water at a silica surface as revealed by vibrational spectroscopy. Langmuir 2010, 26(5), 3407-3414.

13. Li, T.-D.; Gao, J.; Szoszkiewicz, R.; Landman, U.; Riedo, E. Structured and viscous water in subnanometer gaps. Physical Review B 2007, 75, 115415.

14. Miranda, P. B.; Shen, Y. R. Liquid interfaces: a study by sum-frequency vibrational spectroscopy. Journal of Physical Chemistry B 1999, 103, 3292-3307.

15. Du, Q.; Freysz, E.; Shen, Y. R. Surface vibrational spectroscopic studies of hydrogen bonding and hydrophobicity. Science 1994, 264(5160), 826-828.

16. Mezger, M.; Reichert, H.; Schoder, S., et al. Highresolution in situ $\mathrm{X}$-ray study of the hydrophobic gap at the water-octadecyl-trichlorosilane interface. Proceedings of the National Academy of Sciences 2006, 103(49), 18401-18404.
17. Du, H.; Miller, J. D. A molecular dynamics simulation study of water structure and adsorption states at talc surfaces. International Journal of Mineral Processing 2007, 84, 172-184.

18. Eriksson, J. C.; and Yoon, R. H. The nature of hydrophobic attractive forces. In Froth Flotation: A Century of Innovation (Fuerstenau, M. C.; Jameson, G.; Yoon, R. -H. (eds.)), Littleton, Colorado: SME, 2007, 133-177.

19. Nguyen, A. V.; Schulze, H. J.; Ralston, J. Elementary steps in particle-bubble attachment. International Journal of Mineral Processing 1997, 51, 183-195.

20. Shen, Y.; Satoshi, N.; Kohei, U. Sum frequency generation (SFG) study of the pH-dependent water structure on a fused quartz surface modified by an octadecyltrichlorosilane (OTS) monolayer. Physical Chemistry Chemical Physics 2001, 3, 3463-3469.

21. Israelachvili, J. N. The calculation of van de Waals dispersion forces between macroscopic bodies. Proceedings Royal Society of London A 1972, 331(1584), 39-55.

22. Laskowski, J. The relationship between flotability and hydrophobicity. In Advances in Mineral Processing, Arbiter Symposium, Littleton, Colorado: SME, 1986, chapter $11,196$.

\section{WHAT DO YOU THINK?}

To discuss this paper, please email up to 500 words to the managing editor at sufi@icepublishing.com

Your contribution will be forwarded to the author(s) for a reply and, if considered appropriate by the editor-inchief, will be published as a discussion in a future issue of the journal.

ICE Science journals rely entirely on contributions sent in by professionals, academics and students coming from the field of materials science and engineering. Articles should be within 5000-7000 words long (short communications and opinion articles should be within 2000 words long), with adequate illustrations and references. To access our author guidelines and how to submit your paper, please refer to the journal website at www.icevirtuallibrary.com/sufi 